Corresponding authors:

Wanxue.xu@mgh.harvard.edu; Rbalasubramanian

@mgh.harvard.edu

(c) $2020 \mathrm{Xu}$ et al. This article is distributed under the terms of the Creative Commons Attribution-NonCommercial License, which permits reuse and redistribution, except for commercial purposes, provided that the original author and source are credited.

Ontology term: hypothalamic gonadotropin-releasing hormone (GNRH) deficiency

Published by Cold Spring Harbor Laboratory Press

doi:10.1101/mcs.a005033

\section{Hypogonadotropic hypogonadism due to variants in RAB3GAP2: expanding the phenotypic and genotypic spectrum of Martsolf syndrome}

\author{
Wanxue Xu, ${ }_{1}^{1}$ Lacey Plummer, ${ }^{1}$ Richard Quinton, ${ }^{2}$ Francesca Swords, ${ }^{3}$ William \\ F. Crowley, ${ }^{1}$ Stephanie B. Seminara, ${ }^{1}$ and Ravikumar Balasubramanian ${ }^{1}$ \\ ${ }^{1}$ Harvard Reproductive Endocrine Sciences Center, Reproductive Endocrine Unit of the Department of \\ Medicine, Massachusetts General Hospital, Boston, Massachusetts 02114, USA; ${ }^{2}$ Newcastle-upon-Tyne \\ Hospitals Foundation NHS Trust (Royal Victoria Infirmary) and Institute of Genetic Medicine, University of \\ Newcastle-upon-Tyne, Newcastle-upon-Tyne NE1 7RU, United Kingdom; ${ }^{3}$ Clinical Research and Trials Unit, \\ Norfolk and Norwich University Hospitals NHS Foundation Trust, Norwich NR4 7UY, United Kingdom
}

Abstract Biallelic pathogenic variants in RAB3GAP2 cause Warburg Micro syndrome (WARBM) and Martsolf syndrome (MS), two rare, phenotypically overlapping disorders characterized by congenital cataracts, intellectual disability, and hypogonadism. Although the initial report documented hypergonadotropic hypogonadism (implying a gonadal defect), an adolescent girl with WARBM/MS was subsequently reported to have hypogonadotropic hypogonadism (implying a central defect in either the hypothalamus or anterior pituitary). However, in adult MS, hypogonadotropism has not been convincingly demonstrated. Additionally, the correlation between the pathogenic severity of variants in RAB3GAP2 and the phenotypic severity also remains unclear. Here we present a clinical report of a woman with congenital cataracts, apparent intellectual disability, and pubertal failure who underwent exome sequencing (ES) to determine a precise molecular diagnosis. Reproductive phenotypes reported previously in individuals with MS and the genotypic spectrum of previous RAB3GAP2 variants were also reviewed. The ES identified pathogenic compound heterozygous RAB3GAP2 variants (c.387-2A > G; p. (Arg428Glu)) combined with her phenotypic features, which enabled a unifying molecular diagnosis of MS. Reproductive evaluation confirmed a normosmic idiopathic hypogonadotropic hypogonadism. Review of the RAB3GAP2 allelic spectrum in WARBM/MS suggests that although variants resulting in complete abrogation of RAB3GAP2 protein function cause severe WARBM, variants associated with partially preserved RAB3GAP2 function cause milder MS. This report expands the genotypic and phenotypic spectrum of MS and demonstrates hypogonadotropic hypogonadism as a key pathophysiologic abnormality in MS. Genotype-phenotype associations of previously reported RAB3GAP2 variants indicate that variants that fully abolish RAB3GAP2 function result in WARBM, whereas MS is associated with variants of lesser severity with residual RAB3GAP2 function.

[Supplemental material is available for this article.]

\section{INTRODUCTION}

Warburg Micro syndrome (WARBM; MIM614225) and Martsolf syndrome (MS; MIM212720) represent the severe and mild forms, respectively, of a clinical syndrome characterized by the 
association of congenital cataracts, microcephaly, intellecutual disability, and hypogonadism (Aligianis et al. 2006; Borck et al. 2011). Compared to the WARBM phenotype, individuals with MS lack optic atrophy; have less severe functional visual impairment; do not have polymicrogyria or corpus callosal defects on brain imaging; have milder intellectual disability; and have muscular spasticity restricted to their lower limbs. Four genes (RAB3GAP1, RAB3GAP2, RAB18, and TBC1D20) are associated with WARBM/MS. Pathogenic variants in these genes result in dysfunctions of a RAB18, a GTPase, either directly (RAB18) or indirectly (RAB3GAP1, RAB3GAP2, TBC1D20) (Handley et al. 2013; Handley and Sheridan 2018) and in RAB18 dysregulation and are thus believed to contribute to the disease pathology in both WARBM and MS via disruptions of these biologic pathways.

Although hypogonadism is a key feature in the WARBM/MS spectrum, most reproductive phenotypes in individuals with WARBM/MS (Sánchez et al. 1985; Hennekam et al. 1988; Harbord et al. 1989; Aligianis et al. 2006; Handley et al. 2013; Gumus 2018; Handley and Sheridan 2018) have only been ascertained prepubertally (i.e., in infancy or prepuberty) and longitudinal studies regarding pubertal transition in these patients are also lacking. In addition, there is contradictory information regarding the nature of the hypogonadism (i.e., primary vs. secondary) in the handful of clinical reports involving adolescent/ adult patients. In the initial report, two adult males had hypergonadotropism but with normal serum testosterone levels, suggestive of a compensated state of primary hypogonadism (i.e., a gonadal defect) (Martsolf et al. 1978). In contrast, a 17-yr-old prepubertal female with MS and primary amenorrhea was subsequently reported who had hypogonadotropic hypogonadism (Hennekam et al. 1988), suggesting secondary hypogonadism (i.e., a defect in hypothalamus and/or anterior pituitary). Unfortunately, genetic studies were not included in these prior reports. Herein, we describe a genotype-guided study that identified novel pathogenic variants in RAB3GAP2 in an adult patient fulfilling clinical criteria for MS with complete absence of puberty and hypogonadotropic hypogonadism (suggesting underlying hypothalamic and/or pituitary defect) associated with RAB3GAP2-related MS.

\section{RESULTS}

\section{Clinical Presentation and Family History}

A female proband, born to nonconsanguineous parents, presented at $2 \mathrm{wk}$ of age with bilateral cataracts and underwent lensectomies and subsequent strabismus repair. In late infancy, she was also noted to have hearing difficulties. Initially, the proband attended a school for visually impaired and, upon recognition of moderate learning difficulties, was transferred to a school for special education needs. Although she showed steady linear growth, she remained shorter than her peers throughout childhood. Other developmental milestones were also significantly delayed (walking at $21 / 2 \mathrm{yr}$ of age, speech initiation at $3 \mathrm{yr}$ of age). Auditory testing showed low tone hearing loss (1000-6000 Hz-audible at threshold 20-30 dB, but reduced hearing at 250 and $500 \mathrm{~Hz}$-audible at threshold 50-60 dB). At $17 \mathrm{yr}$ of age, she presented to her endocrinologist with absent thelarche and menarche. She reported a normal sense of smell, and her physical examination revealed bilateral clinodactyly (radial, F5) and bilateral cutaneous syndactyly (partial fusion, T2-3). She had Tanner II axillary hair and Tanner IV pubic hair. Biochemical evaluation confirmed hypogonadotropic hypogonadism (LH: $0.2 \mathrm{IU} / \mathrm{L} ; \mathrm{FSH}: 0.8 \mathrm{IU} / \mathrm{L}$; estradiol [ $\mathrm{E}_{2}$ ]: $49 \mathrm{pmol} / \mathrm{L}$ [reference range: 55-1284 pmol/L]) with otherwise normal baseline anterior pituitary functions (prolactin, thyroid function tests, cortisol, and IGF-1). A cranial MRI showed a normal pituitary and intact olfactory structures. No polymicrogyria was noted and her corpus callosum was normal. Pelvic ultrasound showed a $<1-\mathrm{cm}$, rudimentary uterus and small ovaries; findings were consistent with an endocrine diagnosis of normosmic idiopathic hypogonadotropic hypogonadism ( $\mathrm{nlHH}$ ) 
(Seminara and Crowley 2002). After clinical evaluation, sex steroid replacement was initiated, and the patient responded with appearance of secondary sexual characteristics, a pubertal growth spurt, and the onset of menses. Her mother had normal menarche at $12 \mathrm{yr}$ of age. Although the patient was referred to our research program at age of $20 \mathrm{yr}$, this clinical report was initiated upon availability of her exome sequencing (ES) results that were performed more recently. The ES data were available from the index patient and her mother (Fig. 1). Clinical information and DNA samples from her father and elder sibling were not available.

Literature Review of Reproductive Phenotypes in Previously Reported MS Individuals First, we reviewed genotypes and phenotypes of previously reported individuals with MS with RAB3GAP2 pathogenic variants (Table 1). Nine patients (three males, six females; from four families) were identified (Table 1). Reproductive phenotypes were available in five individuals (three males and two females). Additionally, treatment and olfactory impairment were not reported in the reviewed literature. All three males were prepubertal at the time of report, but each had evidence of neonatal hypogonadism during the neonatal period (i.e., micropenis and/or cryptorchidism). Intriguingly, the reproductive phenotypes noted in the two female patients (ages $17 \mathrm{yr}$ and $14 \mathrm{yr}$ ) were reportedly normal. The genotypic spectrum consisted of three homozygous pathogenic missense variants and one homozygous splice-altering variant. In addition to these genetically defined individuals with MS, we also reviewed reproductive phenotypes reported in four patients (three males; one female) without a genetic diagnosis (Supplemental Table 2). Two adult males were reported in the initial report by Martsolf et al. (1978), both of whom had small testes and clinical testing showed elevated gonadotropins but with normal testosterone levels (i.e., compensated hypergonadotropic hypogonadism). Hennekam et al. (1988) reported two patients:

A

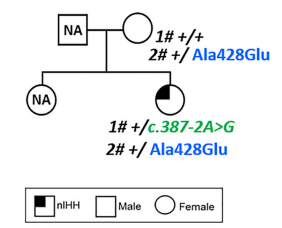

B

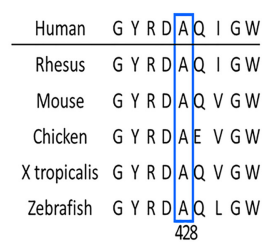

C

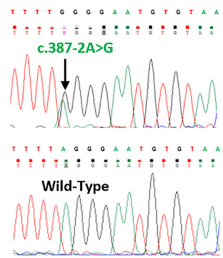

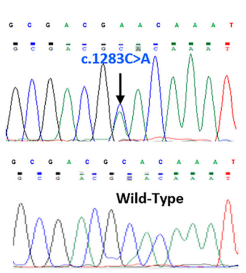

D

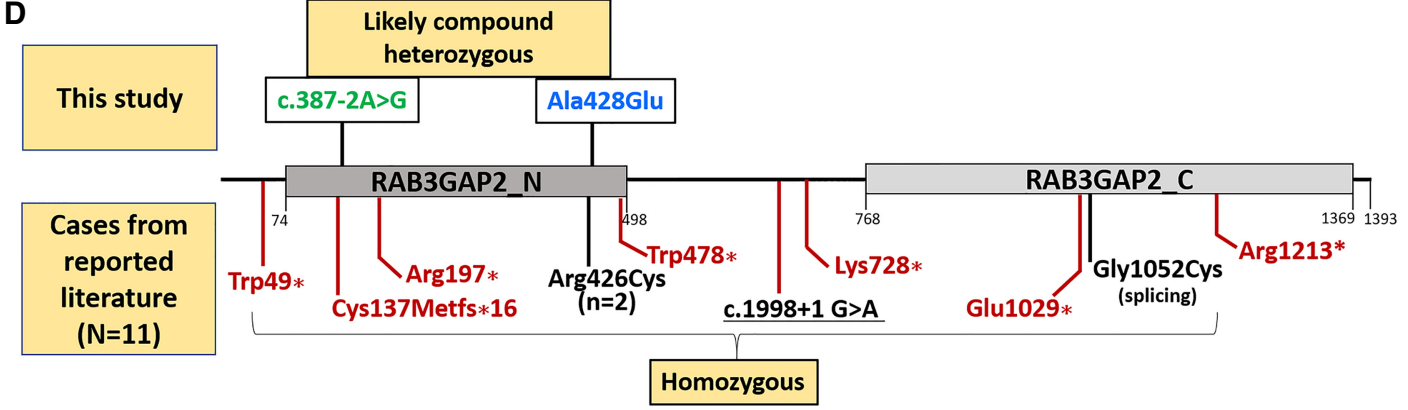

Figure 1. Pedigree, multiple species alignments of RAB3GAP2 protein, electropherogram, and RAB3GAP2 variants identified to date in WARBM/MS. (A) Pedigree of index patient and segregation of RAB3GAP2 variants identified in this report; $(B)$ multispecies protein conservation of novel missense variant ( $p$.Arg428Glu) found in index individual showing conservation from humans to zebrafish; $(C)$ electropherograms of variants identified in this study; and (D) schematic of RAB3GAP2 protein showing MS-associated RAB3GAP2 variants identified in this report (shown above protein schematic; in green and blue) and RAB3GAP2 variants reported in the literature (shown below protein schematic). (Red) WARBM-associated variants, (black) MS-associated variants. The RAB3GAP2 protein domains were defined using Interpro (Mitchell et al. 2019) and Pfam (El-Gebali et al. 2019) resources. 


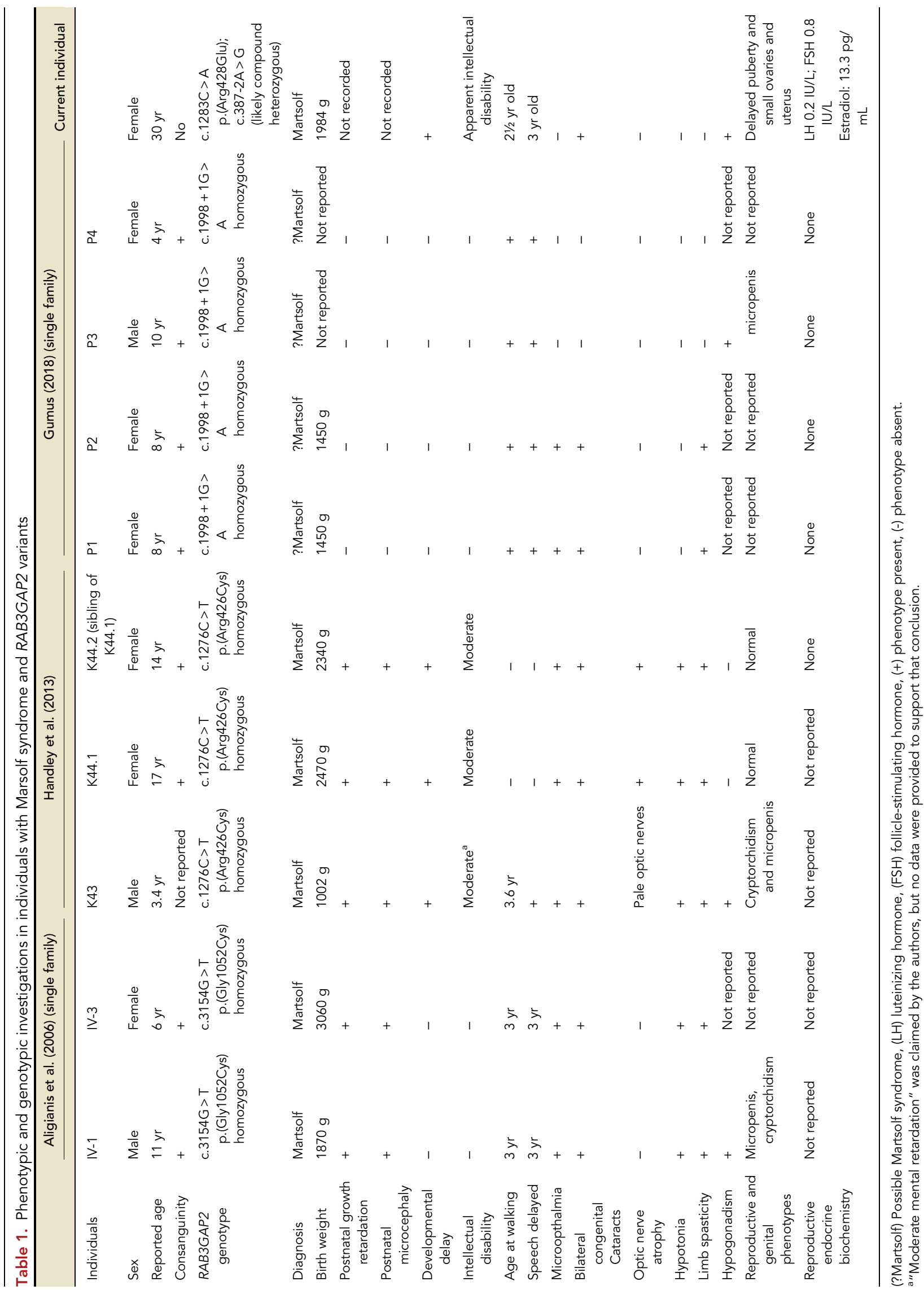


a prepubertal boy (age $12 \mathrm{yr}$ ) with micropenis and cryptorchidism and a 17-yr-old female with amenorrhea and biochemical evidence of hypogonadotropic hypogonadism.

\section{Exome Sequencing Analysis and Review of the RAB3GAP2 Allelic Spectrum in WARBM/MS}

The ES data from the index patient were reviewed for known genes reported to cause idiopathic hypogonadotropic hypogonadism including genes reported to cause syndromic forms of IHH (Supplemental Table 1). This analysis identified a c.387-2A $>\mathrm{G}$ variant in RAB3GAP2 (NM_012414.3). This variant had a frequency of $4.1 \times 10^{-06}$ in gnomAD and four independent splice prediction programs (Berkeley Drosophila Genome Project [BDGP], Alternative Splice Site Predictor [ASSP], Mutation Taster, and Human Splicing Finder) categorized this variant as deleterious secondary to a splicing defect (Table 2). The proband was also found to harbor a c.1283C > A variant in RAB3GAP2, which predicts p.(Ala428Glu) (Fig. 1A,B). This variant was novel, disrupted a highly conserved amino acid residue, and predicted as either deleterious/damaging by multiple prediction programs (SIFT, PolyPhen, CADD, and REVEL) (Table 2). The splice acceptor (c.387-2A > G) was graded as pathogenic and the missense variant p.(Arg428Glu) was graded as likely pathogenic using American College of Medical Genetics (ACMG) guidlines (Richards et al. 2015). In addition, the proband did not harbor any variants in the coding sequence of other known IHH genes (Supplemental Table 1). Although her paternal DNA sample was not available for segregation analysis, her mother shared the c.1283C > A p.(Arg428Glu) likely pathogenic variant but did not harbor the pathogenic splice variant. The likelihood of the essential splicing variant arising de novo on the maternal haplotype in cis with the p.(Arg428Glu) variant was considered. However, we did not perform additional experiments (cell cloning/ long-range polymerase chain reaction [PCR]) as we deemed that this scenario was unlikely given the previous clear association of biallelic RAB3GAP2 pathogenic variants with MS. Hence, it was presumed that the variants were most likely to have been inherited in the compound heterozygous state (Fig. 1C).

We also cataloged all previously reported RAB3GAP2 variants in WARBM/MS (Fig. 1D). Notably, all WARBM-associated RAB3GAP2 variants were protein-truncating mutations that have been shown to completely abolish RAB3GAP2 function (Handley et al. 2013; Handley and Sheridan 2018). In contrast, including this report, all MS-associated RAB3GAP2 variants were predominantly missense variants or splicing variants that have been deemed to reduce, but not fully abolish, RAB3GAP2 function.

\section{DISCUSSION}

This clinical report highlights the index patient's clinical odyssey involving multiple diagnostic evaluations without an accurate and timely diagnosis, typifying the clinical challenges posed to both patients and physicians by such rare clinical syndromes. She presented in infancy to ophthalmology with congenital cataracts, to pediatrics in childhood with apparent intellectual disability, and subsequently to endocrinology in adolescence with absent puberty. Eventually, ES identified the two rare variants (pathogenic/likely pathogenic, respectively) in RAB3GAP2, a gene previously associated wth the WARBM/MS spectrum (Martsolf et al. 1978; Warburg et al. 1993). The proband had milder phenotypes across the fuller WARBM/MS spectrum, and hence a diagnosis of RAB3GAP2-related MS was made several years after her initial clinical presentation, attesting to the utility of genetic testing in providing a unifying clinical diagnosis in individuals with rare syndromic disorders. Furthermore, this study is the first longitudinal description of the reproductive phenotypes (pubertal presentation and biochemical investigations) in an individual with RAB3GAP2-associated MS 


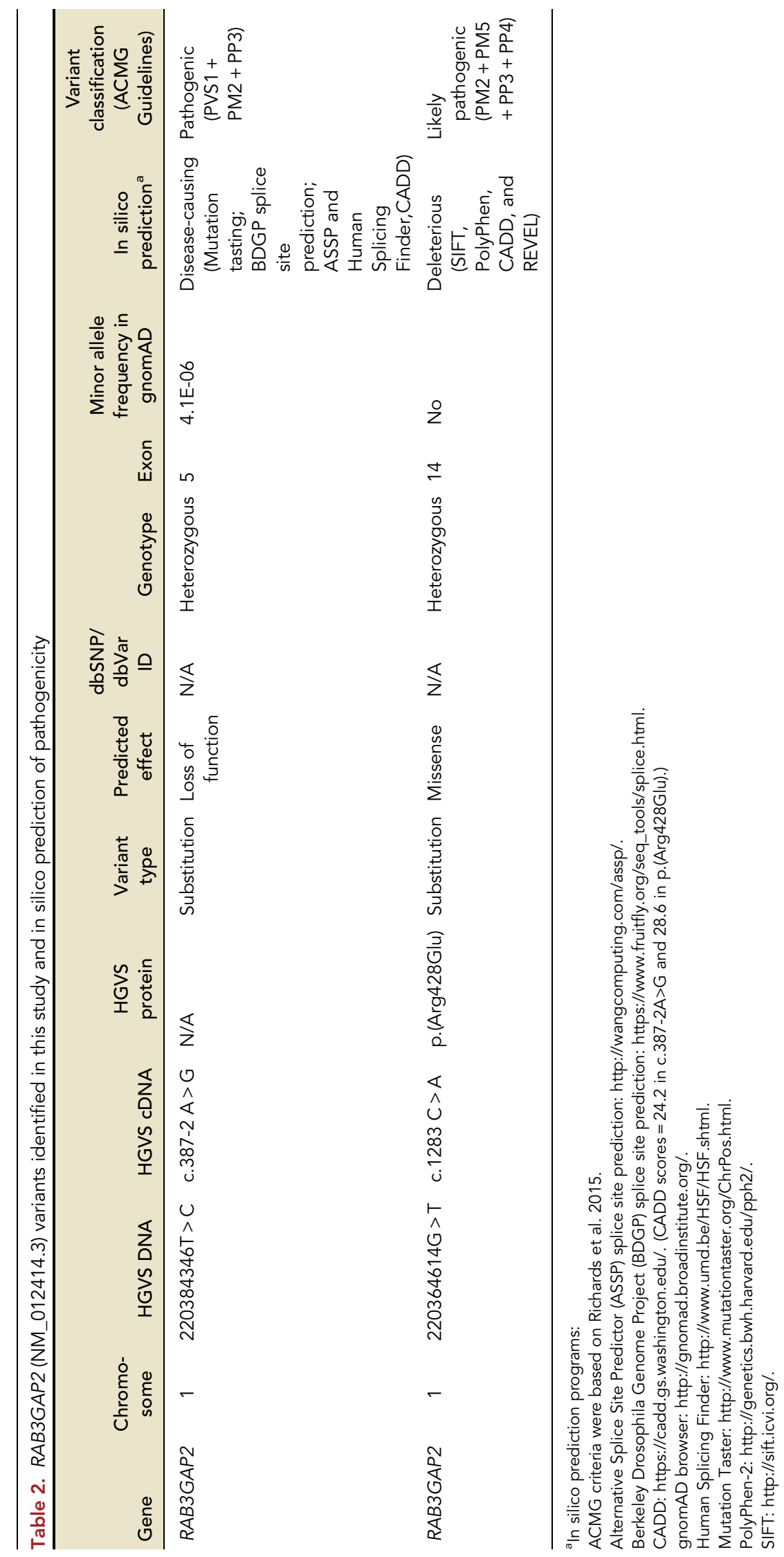


wherein we establish hypogonadotropic hypogonadism as a key pathophysiologic defect in this rare syndrome.

RAB3GAP2 is a GTPase-activating protein, known to form a complex with RAB3GAP1 that together regulates the GTP hydrolysis activity of the Rab protein family of small GTPase(s) with putative exocytotic roles (Handley and Aligianis 2012). Our observations that RAB3GAP2-related MS is associated with hypogonadotropic hypogonadism are consistent with the previously reported interaction of RAB3GAP2 with rabconnectin 3a, a protein expressed in exocytosis vesicles in gonadotropin-releasing hormone $(\mathrm{GnRH})$ axonal terminals in the median eminence of the hypothalamus and in the cells expressing luteinizing hormone (LH) and follicle-stimulating hormone (FSH) within the anterior pituitary gonadotropin-producing cells (Tata et al. 2014, 2017). Therefore, we hypothesize that RAB3GAP2-associated hypogonadotropic hypogonadism may also result from defects in hormone release at the hypothalamic and/or pituitary levels. In that regard, it is notable that the first reported individual with MS had hyper- (rather than hypo-) gonadotropic hypogonadism, implicating a primary gonadal defect. To fully establish the precise site of the deficit(s) (hypothalamic vs. pituitary with or without additional gonadal defects) in RAB3GAP2-related WARBM/MS, detailed neuroendocrine studies with GnRH administration will be required, but this was not available for the patient described in this study. In this regard, it is notable that some genetic pathways already implicated as causing reproductive disorders are known to disrupt function at multiple levels across the hypothalamo-pituitary-gonadal axes, resulting in both hyperand hypogonadotropic phenotypes (e.g., NROB1/DAX1, Prader-Willi locus, congenital myotonic dystrophy) (Hamilton et al. 1972; Febres et al. 1975; Muscatelli et al. 1994; Jadhav et al. 2011; Angulo et al. 2015). Therefore, further longitudinal studies of the reproductive phenotypes along with neuroendocrine studies will be required to fully ascertain the spectrum of reproductive deficits in WARBM/MS.

It has been hypothesized that the severity of the underlying RAB3GAP2 pathogenic variants (Handley and Aligianis 2012; Handley et al. 2013; Handley and Sheridan 2018) may determine the resultant clinical phenotypes (severe WARBM vs. milder MS). To date, all reported RAB3GAP2-related WARBM individuals have harbored homozygous, inactivating variants (frameshifting indels or stop variants). In contrast, the RAB3GAP2-related MS genotypic spectrum includes two distinct homozygous missense variants and one homozygous splice-site variant (Table 1; Fig. 1D). One of the MS-associated missense mutations (p.Gly1052Cys) was shown to disrupt splicing but produced some preserved full-length protein with likely residual protein function (Aligianis et al. 2006). Similarly, the second MS-associated RAB3GAP2 missense variant (p.Arg426Cys) was also deemed not to completely disrupt RAB3GAP2 protein function (Handley et al. 2013). Experimental data on the precise consequence of the MS-associated splice variant have not been reported (Gumus 2018). The MS-associated missense mutation identified in this report (p.Ala428Glu) affects a conserved amino acid residue, is predicted to be likely pathogenic by ACMG criteria, occurs close to a previously reported MS-associated variant (p.Arg426Cys), and hence is likely to have reduced functional activity without full abrogation of protein function. The essential splice acceptor variant (c.387-2A $>\mathrm{G}$ ) identified in this report is predicted to result in an in-frame deletion (p.Glu130_Arg145del) secondary to skipping of exon 5 of the RAB3GAP2 gene, with some retained protein function. Thus, in contrast to severe WARBM-associated RAB3GAP2 variants, almost all of the MS-associated RAB3GAP2 variants (including this report) retain some residual protein function, and these findings lend further support to prior observations that the severity of the variants underlie genotype-phenotype correlations (Handley and Aligianis 2012; Handley et al. 2013; Handley and Sheridan 2018). However, as shown in Table 1, expressivity is still variable across families harboring the less severe RAB3GAP2 variants, which suggests that there may be other genetic/nongenetic modifiers that determine the clinical phenotype. In this regard, because our ES analysis was restricted 
to genes linked primarily to idiopathic hypogonadotropic hypogonadism, the potential contribution of other genes to the variable phenotypic expressivity could not be examined, and this represents a limitation of this current study.

A key challenge in accurate and timely diagnosis of complex syndromes relates to "temporal gaps" in the recognition of constituent phenotypes that become evident across the life span at various developmental stages. For example, in WARBM/MS, congenital cataracts and neurological features (developmental delay, intellectual disability, and spasticity) are recognized in early in childhood, whereas reproductive phenotypes are typically not readily evident in childhood. In this regard, our clinical observation that WARBM/MS is characterized by hypogonadotropic hypogonadism is particularly important. It is now established that the reproductive cascade is paradoxically active in the first few months of life in both sexes (Grumbach 2005). During the first $6 \mathrm{mo}$ in boys and $\sim 12 \mathrm{mo}$ in girls, sex-steroid and gonadotropin levels are equivalent to those observed in adulthood, and this developmental window is referred to as the minipuberty of infancy (Dunkel and Quinton 2014). Micropenis and/or cryptorchidism (undescended testes) in boys may be signs of absent minipuberty in boys and laboratory assessment of gonadotropin levels at this developmental window may indicate hypogonadotropic hypogonadism. Indeed, in all of the males with RAB3GAP2-related MS, evidence of a defective minipuberty was readily evident (micropenis/cryptorchidism) and, thus, in conjunction with other clinical signs, a diagnosis of MS was feasible (Table 1). Furthermore, recognition of hypogonadotropic hypogonadism in boys also allows the administration of neonatal testosterone/hCG therapy to help promote penile growth and alert the observant physician about the need for diligent prospective endocrine evaluation during adolescence. This recognition helps timely initiation of secondary sexual characteristics, optimizes adult height/bone health, and avoids much of the psychological sequalae of delayed puberty. In sharp contrast to boys, disruption of minipuberty in girls is typically not recognizable as there are no physical signs that are readily evident in infant girls. Indeed, in the patient described in this report, although ocular/neurological phenotypes were recognized early, a diagnosis of WARBM/MS was not feasible because hypogonadism, a key defining feature of WARBM/MS, could only be demonstrable at adolescence when pubertal failure became evident. Hence, in girls presenting with ocular and neurological signs of WARBM/MS (and in boys without discernible micropenis/cryptorchidism), genetic testing must be considered to allow an earlier molecular diagnosis of the WARBM/ MS spectrum that will facilitate prospective endocrine evaluations and multidisciplinary involvement including optimal endocrine care and timely initiation of sex-steroid therapy.

In summary, this report illustrates the emerging importance of human genetics and nextgeneration sequencing in providing a unifying clinical and molecular diagnosis in rare syndromic presentations of $\mathrm{IHH}$. These combined approaches additionally provide a unique opportunity to catalog the mutational and phenotypic spectrum of these syndromes and inform the pathophysiology, clinical care, and recurrence risk assessments.

\section{METHODS}

This research was approved by the Partners Institutional Review Board at the Massachusetts General Hospital. The family participated in this study after their informed consent was obtained.

\section{Genetic Analysis}

ES was performed at The Broad Institute Genomics Platform. Variant calling was performed with GATK creating gVCF files for each sample that were then jointly called using the GATK Genotype gVCF module. Variant quality scores were derived using GATK best practices for 
Table 3. Exome sequencing coverage table for the RAB3GAP2

\begin{tabular}{lccc}
\hline Sample & $\begin{array}{c}\text { Percentage of reads } \\
\text { aligned }\end{array}$ & $\begin{array}{c}\text { Average read } \\
\text { coverage }\end{array}$ & $\begin{array}{c}\text { Percentage of RAB3GAP2 sites with 10X } \\
\text { coverage }\end{array}$ \\
\hline Proband & $94 \%$ & 136.5 & $99 \%$ \\
\hline
\end{tabular}

indels and single-nucleotide variants. The jointly called VCF files were then analyzed using the integrative database framework, GEMINI v.0.19.1. Variant annotation was performed with the Ensembl Variant Effect Predictor (VEP) against the GRC37/hg19 reference Human Genome. Rare sequence variants (RSVs) for both homozygous and heterozygous RSVs were based on a minor allele frequency (MAF) of $<0.1 \%$ as determined by the genome Aggregation Database (gnomAD) browser (Karczewski et al. 2019). All RSVs were confirmed in bidirectional Sanger sequencing in all probands and their pattern of segregation established in all available family members. ES coverage for the RAB3GAP2 is shown in Table 3. Sanger sequencing was performed by the Center for Computational and Integrative Biology (CCIB) DNA Core Facility at Massachusetts General Hospital (Cambridge, MA). The ES data from the index patient were reviewed for known genes associated with syndromic and nonsyndromic IHH (Supplemental Table 1). The protein domains were defined using InterPro (Mitchell et al. 2019) and Pfam (El-Gebali et al. 2019) resources.

\section{Literature Review}

PubMed was searched for original full-text articles (English language) published up to November 2019 discussing Martsolf syndrome and RAB3GAP2-associated WARBM/MS. The search keywords included "Martsolf syndrome," "Warburg-Micro syndrome," "phenotype," "genotype," "RAB3GAP2," "genetics," "gene mutation," and "variant." Relevant articles of individuals with MS, genotypes, and endocrine phenotypes were collated.

\section{ADDITIONAL INFORMATION}

\section{Data Deposition and Access}

All sequence data and interpreted variants have been deposited in ClinVar (https://www.ncbi .nlm.nih.gov/clinvar/) under accession numbers SCV001245468 and SCV001245469.

\section{Ethics Statement}

Written informed consent was obtained for all individuals in this study. The study was approved by the Partners Institutional Review Board of Massachusetts General Hospital,

Competing Interest Statement

The authors have declared no competing interest.

Referees

Chris Gunter

Mustafa Ozen

Anonymous

Received December 4, 2019; accepted in revised form February 13, 2020.
Boston, MA (under protocol \#1999P006955).

\section{Acknowledgments}

The authors thank the family members for their participation in this study.

\section{Author Contributions}

W.F.C., R.B., and W.X. conceived the study and its design. R.Q., F.S., W.X., L.P., and R.B. acquired, analyzed, and interpreted the data. W.X., S.B.S., and R.B. came up with the conceptual outline for the article. W.X. and R.B. drafted the manuscript. S.B.S., W.F.C., and R.B. critically revised the manuscript draft. All authors have approved the current version of the manuscript and its submission to Cold Spring Harbor Molecular Case Studies. 


\section{Funding}

This report was supported by grants from the National Institutes of Child Health and Development: HD028138 to R.B., W.F.C., L.P., and W.F.C., HD096324 to R.B., HD077043 to R.B., and HD043341 to S.B.S.

\section{REFERENCES}

Aligianis IA, Morgan NV, Mione M, Johnson CA, Rosser E, Hennekam RC, Adams G, Trembath RC, Pilz DT, Stoodley N, et al. 2006. Mutation in Rab3 GTPase-activating protein (RAB3GAP) noncatalytic subunit in a kindred with Martsolf syndrome. Am J Hum Genet 78: 702-707. doi:10.1086/502681

Angulo MA, Butler MG, Cataletto ME. 2015. Prader-Willi syndrome: a review of clinical, genetic, and endocrine findings. J Endocrinol Invest 38: 1249-1263. doi:10.1007/s40618-015-0312-9

Borck G, Wunram H, Steiert A, Volk AE, Körber F, Roters S, Herkenrath P, Wollnik B, Morris-Rosendahl DJ, Kubisch C. 2011. A homozygous RAB3GAP2 mutation causes Warburg Micro syndrome. Hum Genet 129: 45-50. doi:10.1007/s00439-010-0896-2

Dunkel L, Quinton R. 2014. Transition in endocrinology: induction of puberty. Eur J Endocrinol 170: R229R239. doi:10.1530/EJE-13-0894

El-Gebali S, Mistry J, Bateman A, Eddy SR, Luciani A, Potter SC, Qureshi M, Richardson LJ, Salazar GA, Smart A, et al. 2019. The Pfam protein families database in 2019. Nucleic Acids Res 47: D427-D432. doi:10.1093/ nar/gky995

Febres F, Scaglia H, Lisker R, Espinosa J, Morato T, Shkurovich M, Pérez-Palacios G. 1975. Hypothalamic-pituitary-gonadal function in patients with myotonic dystrophy. J Clin Endocrinol Metab 41: 833-840. doi:10 $.1210 /$ jcem-41-5-833

Grumbach MM. 2005. A window of opportunity: the diagnosis of gonadotropin deficiency in the male infant. J Clin Endocrinol Metab 90: 3122-3127. doi:10.1210/jc.2004-2465

Gumus E. 2018. Case report of four siblings in southeast Turkey with a novel RAB3GAP2 splice site mutation: Warburg Micro syndrome or Martsolf syndrome? Ophthalmic Genet 39: 391-395. doi:10.1080/13816810 .2018.1432065

Hamilton CR Jr, Scully RE, Kliman B. 1972. Hypogonadotropinism in Prader-Willi syndrome. Induction of puberty and sperm altogenesis by clomiphene citrate. Am J Med 52: 322-329. doi:10.1016/0002-9343(72)90019-8

Handley MT, Aligianis IA. 2012. RAB3GAP1, RAB3GAP2 and RAB18: disease genes in Micro and Martsolf syndromes. Biochem Soc Trans 40: 1394-1397. doi:10.1042/BST20120169

Handley, M., and E. Sheridan. 2018. RAB18 deficiency. In GeneReviews ${ }^{\circledR}$ (ed. Adam MP, Ardinger HH, Pagon RA, et al.). University of Washington, Seattle, WA. https://www.ncbi.nlm.nih.gov/books/NBK475670

Handley MT, Morris-Rosendahl DJ, Brown S, Macdonald F, Hardy C, Bem D, Carpanini SM, Borck G, Martorell L, Izzi C, et al. 2013. Mutation spectrum in RAB3GAP1, RAB3GAP2, and RAB18 and genotype-phenotype correlations in Warburg Micro syndrome and Martsolf syndrome. Hum Mutat 34: 686-696. doi:10.1002/ humu.22296

Harbord MG, Baraitser M, Wilson J. 1989. Microcephaly, mental retardation, cataracts, and hypogonadism in sibs: Martsolf's syndrome. J Med Genet 26: 397-400. doi:10.1136/jmg.26.6.397

Hennekam RC, van de Meeberg AG, van Doorne JM, Dijkstra PF, Bijlsma JB. 1988. Martsolf syndrome in a brother and sister: clinical features and pattern of inheritance. Eur J Pediatr 147: 539-543. doi:10.1007/ BF00441986

Jadhav U, Harris RM, Jameson JL. 2011. Hypogonadotropic hypogonadism in subjects with DAX1 mutations. Mol Cell Endocrinol 346: 65-73. doi:10.1016/j.mce.2011.04.017

Karczewski KJ, Francioli LC, Tiao G, Cummings BB, Alföldi J, Wang Q, Collins RL, Laricchia KM, Ganna A Birnbaum DP, et al. 2019. Variation across 141,456 human exomes and genomes reveals the spectrum of loss-of-function intolerance across human protein-coding genes. bioRxiv doi:10.1101/531210

Martsolf JT, Hunter AG, Haworth JC. 1978. Severe mental retardation, cataracts, short stature, and primary hypogonadism in two brothers. Am J Med Genet 1: 291-299. doi:10.1002/ajmg.1320010305

Mitchell AL, Attwood TK, Babbitt PC, Blum M, Bork P, Bridge A, Brown SD, Chang HY, El-Gebali S, Fraser MI, et al. 2019. InterPro in 2019: improving coverage, classification and access to protein sequence annotations. Nucleic Acids Res 47: D351-D360. doi:10.1093/nar/gky1100

Muscatelli F, Strom TM, Walker AP, Zanaria E, Recan D, Meindl A, Bardoni B, Guioli S, Zehetner G, Rabl W et al. 1994. Mutations in the DAX-1 gene give rise to both X-linked adrenal hypoplasia congenita and hypogonadotropic hypogonadism. Nature 372: 672-676. doi:10.1038/372672a0 
Richards S, Aziz N, Bale S, Bick D, Das S, Gastier-Foster J, Grody WW, Hegde M, Lyon E, Spector E, et al. 2015. Standards and guidelines for the interpretation of sequence variants: a joint consensus recommendation of the American College of Medical Genetics and Genomics and the Association for Molecular Pathology. Genet Med 17: 405-424.

Sanchez JM, Barreiro C, Freilij H. 1985. Two brothers with Martsolf's syndrome. J Med Genet 22: 308-310. doi:10.1136/jmg.22.4.308

Seminara SB, Crowley WF Jr. 2002. Genetic approaches to unraveling reproductive disorders: examples of bedside to bench research in the genomic era. Endocr Rev 23: 382-392. doi:10.1210/edrv.23.3.0469

Tata B, Huijbregts L, Jacquier S, Csaba Z, Genin E, Meyer V, Leka S, Dupont J, Charles P, Chevenne D, et al. 2014. Haploinsufficiency of $D m x / 2$, encoding a synaptic protein, causes infertility associated with a loss of GnRH neurons in mouse. PLoS Biol 12: e1001952. doi:10.1371/journal.pbio.1001952

Tata BK, Harbulot C, Csaba Z, Peineau S, Jacquier S, de Roux N. 2017. Rabconnectin-3a is required for the morphological maturation of GnRH neurons and kisspeptin responsiveness. Sci Rep 7: 42463. doi:10 $.1038 /$ srep42463

Warburg M, Sjo O, Fledelius HC, Pedersen SA. 1993. Autosomal recessive microcephaly, microcornea, congenital cataract, mental retardation, optic atrophy, and hypogenitalism. Micro syndrome. Am J Dis Child 147: 1309-1312. doi:10.1001/archpedi.1993.02160360051017 


\section{COLD SPRING HARBOR Molecular Case Studies}

\section{Hypogonadotropic hypogonadism due to variants in RAB3GAP2: expanding the phenotypic and genotypic spectrum of Martsolf syndrome}

Wanxue Xu, Lacey Plummer, Richard Quinton, et al.

Cold Spring Harb Mol Case Stud 2020, 6: a005033 originally published online May 6, 2020

Access the most recent version at doi:10.1101/mcs.a005033
Supplementary http://molecularcasestudies.cshlp.org/content/suppl/2020/05/21/mcs.a005033.D Material C1
References This article cites 24 articles, 5 of which can be accessed free at: http://molecularcasestudies.cshlp.org/content/6/3/a005033.full.html\#ref-list-1
License This article is distributed under the terms of the Creative Commons Attribution-NonCommercial License, which permits reuse and redistribution, except for commercial purposes, provided that the original author and source are credited.
Email Alerting Receive free email alerts when new articles cite this article - sign up in the box at the Service top right corner of the article or click here.

